\title{
ISOLAMENTO E IDENTIFICAÇÃO DE FUNGOS DO GÊNERO Aspergillus spp. DE ÁGUA UTILIZADA NA REABILITAÇÃO DE PINGUINS-DE- MAGALHÃES
}

\section{ISOLATION AND IDENTIFICATION OF Aspergillus spp. FROM THE WATER USED FOR REHABILITATION OF MAGELLANIC PENGUINS}

\author{
Vanice Rodrigues Poester ${ }^{1 *}$ \\ Gabriel Baracy Klafke ${ }^{1}$ \\ Ângela Leitzke Cabana ${ }^{1}$ \\ Andréa Corrado Adornes ${ }^{2}$ \\ Rodolfo Pinho da Silva Filho ${ }^{2}$ \\ Melissa Orzechowski Xavier ${ }^{1}$

\begin{abstract}
${ }^{1}$ Laboratório de Micologia, Universidade Federal do Rio Grande, Rio Grande, RS,Brasil. ${ }^{2}$ Centro de Recuperação de Animais Marinhos - CRAM-FURG, Rio Grande, RS, Brasil.

*Autora para correspondência - vanicerp@gmail.com
\end{abstract}

\section{Resumo}

A aspergilose caracteriza-se por ser a principal causa de mortalidade de pinguins em cativeiro. A infecção pelo gênero Aspergillus ocorre principalmente por via aérea, porém o fungo pode ter dispersão pela água. Neste sentido, este trabalho teve como objetivo avaliar a água do tanque onde os pinguins-de-Magalhães permanecem para reabilitação no Centro de Recuperação de Animais Marinhos de Rio Grande, RS, Brasil, quanto à presença de fungos filamentosos do gênero Aspergillus. As amostras de água foram coletadas semanalmente durante um período de 10 meses e processadas em um período máximo de seis horas utilizando-se a técnica da membrana filtrante, com incubação a $25^{\circ} \mathrm{C}$ e $37{ }^{\circ} \mathrm{C}$ por até sete dias. Das 40 amostras analisadas, 32 foram positivas para o isolamento do gênero Aspergillus, sendo que dessas $60 \%$ pertenciam à espécie A. fumigatus. Algumas variáveis interferiram significativamente no isolamento do gênero Aspergillus e/ou da espécie A. fumigatus, como temperatura de incubação, sazonalidade e densidade populacional. Este trabalho demonstra que Aspergillus spp. está presente na água, podendo essa ser uma potencial fonte de infecção para os pinguins em reabilitação.

Palavras-chaves: aspergilose; centro de reabilitação; qualidade da água; Spheniscus magellanicus.

\begin{abstract}
Aspergillosis is the main cause of mortality in captivity penguins. The infection occurs mainly by conidia inhalation of the Aspergillus genus, however, the fungus can also be dispersed by water. Therefore, this study aimed to evaluate water quality of the pool where the rehabilitated penguins remain at Centro de Recuperação de Animais Marinhos in Rio Grande city, Brazil, searching for the presence of the fungus Aspergillus spp. Water samples were collected weekly during a ten-month period and processed within six hours, applying the technique of filtrating membrane, with incubation
\end{abstract}


at $25{ }^{\circ} \mathrm{C}$ and $37{ }^{\circ} \mathrm{C}$ during seven days. Of the forty samples analyzed, thirty-two were positive for the presence of Aspergillus genus, from these $60 \%$ correspond to A. fumigatus. Some variables significantly interfered on the isolation of Aspergillus genus and/or Aspergillus fumigatus specie, such as incubation temperature, seasonality and population density. This study showed Aspergillus spp. is present in the water, being one of the possible sources of infections for penguins in rehabilitation.

Keywords: aspergillosis; rehabilitation center; Spheniscus magellanicus; water quality.

Recebido em: 21 fevereiro 2014

Aceito em: 09 junho 2015

\section{Introdução}

A aspergilose é uma infecção fúngica oportunista, primariamente respiratória, causada por fungos do gênero Aspergillus ${ }^{(1,2)}$. Este gênero caracteriza-se por fungos filamentosos com hifas hialinas, septadas e ramificadas em ângulo agudo, as quais se diferenciam em estruturas reprodutivas típicas para dar origem aos conídios ${ }^{(3)}$. Dentro desse gênero, a espécie responsável pelo maior número de casos clínicos tanto em pinguins quanto nos demais hospedeiros é A. fumigatus $^{(4-7)}$, a qual apresenta colônia pulverulenta, verde-azulada e de rápido crescimento ${ }^{(8)}$.

A aspergilose é considerada a maior causa de mortalidade em pinguins em cativeiro ${ }^{(1,3,5,7)}$. No município de Rio Grande, RS, o Centro de Recuperação de Animais Marinhos (CRAM) recebe anualmente de dezenas a centenas de pinguins-de-Magalhães (Spheniscus magellanicus) para reabilitação ${ }^{(9)}$. Muitos destes acabam vindo a óbito por aspergilose, a qual influencia significativamente nas taxas de liberação e reintrodução dos pinguins recebidos em seu habitat natural ${ }^{(9,10)}$.

Os pinguins-de-Magalhães (Spheniscus magellanicus) são aves marinhas, pertencentes à Ordem Sphenisciformes $^{(11)}$, que se reproduzem no sul da costa da América do Sul, nas Ilhas Malvinas e também na Terra do Fogo, no Chile ${ }^{(12)}$. No inverno e na primavera, os pinguins migram em direção ao norte do Brasil para se alimentarem. Durante a migração ficam expostos a adversidades do meio, principalmente à poluição dos oceanos com derivados de óleo ${ }^{(12-15)}$, chegando aos centros de reabilitação debilitados, o que os torna suscetíveis ao desenvolvimento da aspergilose. A debilidade dos animais encaminhados ao CRAM está associada a fatores climáticos e ambientais, como escassez de alimento durante a migração ou intempéries devido a efeito El Niño, ou ainda, a fatores antropológicos como injúrias traumáticas por pesca ou contaminação por derivados de petróleo liberados em alto mar ${ }^{(16,17)}$. A infecção por Aspergillus spp. ocorre principalmente por via aérea, a partir da inalação dos conídios anemófilos infectantes; no entanto, estudos sugerem que a água pode também ser uma importante fonte de infecção ${ }^{(18-22)}$. Devido à grande capacidade de esporulação que estes fungos possuem na água, são capazes de formar micronichos ambientais em um pequeno período de tempo, dificultando a desinfecção do ambiente ${ }^{(22-24)}$. Neste sentido, o estudo objetivou avaliar a qualidade da água do tanque onde os pinguins permanecem para reabilitação no CRAM, quanto à presença de fungos filamentosos do gênero Aspergillus spp.

\section{Material e Métodos}

As coletas foram realizadas semanalmente durante o período de março a dezembro de 2013 no Centro de Recuperação de Animais Marinhos (CRAM) no Museu Oceanográfico "Prof. Eliézer de Carvalho Rios" da Universidade Federal de Rio Grande (FURG), localizado no município de Rio Grande, RS, totalizando 40 amostras, das quais três ocorreram no verão, 13 no outono, 13 no inverno e 11 na primavera.

Foi coletada amostra semanal de água do único tanque onde os pinguins permanecem durante a 
reabilitação no CRAM. As amostras consistiam em um litro de água coletadas em frascos esterilizados, no período da manhã, sendo imediatamente encaminhadas para o Laboratório de Micologia da Faculdade de Medicina da FURG.

As amostras foram processadas em um período máximo de seis horas após a coleta, em cabine de segurança biológica. Foi empregada a técnica de membrana filtrante, utilizando-se membranas de nitrocelulose (Millipore ${ }^{\circledR}$ ), com poros de $0,45 \mu \mathrm{m}$, conforme descrito por Varo et al. ${ }^{(22)}$. Primeiramente, foi realizada a aferição do $\mathrm{pH}$ da água, utilizando-se fitas pH- Fix 0 - 14 (Macherey-Nagel ${ }^{\circledR}$ ). Na sequência, as amostras foram separadas em duas alíquotas de $250 \mathrm{~mL}$, e os $500 \mathrm{~mL}$ restantes foram desprezados. O conteúdo de $250 \mathrm{~mL}$ de cada frasco foi filtrado e, após este processo, as membranas foram divididas em dois fragmentos semelhantes e então subdivididas em seis, resultando em 12 fragmentos. A semeadura destes fragmentos foi realizada em duplicata dispondo seis fragmentos em cada placa de Petri contendo ágar Sabouraud-dextrose (SDA), suplementado com cloranfenicol $(30 \mathrm{mg} / 100 \mathrm{~mL})$. Em seguida, as placas foram incubadas em estufas microbiológicas com temperaturas ajustadas em $25^{\circ} \mathrm{C}$ e $37^{\circ} \mathrm{C}$ por sete dias, sendo avaliadas quanto ao crescimento fúngico a partir do segundo dia. Ao final do período de incubação, foi realizada a contagem de unidades formadoras de colônias (UFC) por amostra coletada.

As colônias isoladas foram subcultivadas pela técnica de esgotamento para obtenção de cultivos puros e posterior identificação, que foi realizada a partir de características macro e micromorfológicas. As características macromorfológicas avaliadas consistiram no tamanho da colônia, características dos bordos, textura, relevo e pigmentação. Já as análises micromorfológicas foram realizadas pela técnica de microcultivo em ágar-batata, visando identificar as estruturas vegetativas e especialmente as estruturas reprodutivas específicas dos gêneros de fungos filamentosos.

As variáveis estudadas consistiram em temperatura de incubação, sendo considerada a média das duplicatas de cada temperatura; $\mathrm{pH}$ da água; estação do ano e densidade populacional. Esta última foi caracterizada pela quantidade de pinguins em reabilitação no tanque no período da coleta, a qual foi categorizada em dois grupos: até cinco animais e acima de cinco.

Os resultados foram analisados com a utilização do programa estatístico SPSS 19.0, utilizando-se o teste de Qui-quadrado, com nível de significância de 95\% $(\mathrm{p}<0,05)$.

\section{Resultados e Discussão}

Das 40 amostras coletadas, $97,5 \%(\mathrm{n}=39)$ foram positivas para o isolamento de fungos filamentosos, totalizando 1028 UFC, sendo 12\% (122 UFC) do gênero Aspergillus isoladas em $80 \%(\mathrm{n}=32)$ das amostras. Dentre as espécies do gênero Aspergillus, foram identificadas as espécies A. niger, A. flavus e A. fumigatus, sendo que esta última representou 61\% (74 UFC) dos isolados de Aspergillus spp. De acordo com Varo et al. ${ }^{(22)}$, a água pode ser uma possível fonte de contaminação para os fungos do gênero Aspergillus, podendo formar micronichos neste ambiente. Esta afirmação corrobora os resultados obtidos, confirmando a presença destes fungos potencialmente patogênicos na água onde os pinguins permanecem para reabilitação no CRAM.

O isolamento de fungos do gênero Aspergillus ocorreu em cerca de 70\% (29/40) e 50\% (21/40) das amostras incubadas a $37^{\circ} \mathrm{C}$ e $25^{\circ} \mathrm{C}$, respectivamente, sem diferença estatística significativa. Porém, a espécie A. fumigatus foi significativamente mais prevalente $(\mathrm{p}=0,043)$ nas amostras incubadas a $37^{\circ} \mathrm{C}$ do que nas amostras incubadas a $25^{\circ} \mathrm{C}$, representando $55 \%(22 / 40)$ e $32,5 \%$ (13/40) de positividade, respectivamente.

A maioria dos fungos são mesófilos, apresentando uma temperatura ótima de crescimento entre $20^{\circ} \mathrm{C}$ e $30^{\circ} \mathrm{C}$. No entanto, a termotolerância que permite que os microrganismos germinem a temperaturas mais elevadas (acima de $37^{\circ} \mathrm{C}$ ) é uma característica do gênero Aspergillus ${ }^{(25-27)}$, o que explica a maior prevalência encontrada neste estudo de isolamento deste microrganismo à temperatura de $37^{\circ} \mathrm{C} \mathrm{em}$ 
relação a $25^{\circ} \mathrm{C}$. A $25{ }^{\circ} \mathrm{C}$ há um maior número de isolados fúngicos de distintos gêneros e espécies, o que aumenta a competitividade entre eles e dificulta o isolamento de um gênero específico ${ }^{(27)}$.

Segundo Latgé(25) a espécie A. fumigatus apresenta termotolerância mais elevada que as outras espécies do gênero, o que condiz com as informações encontradas nesta pesquisa ${ }^{(27)}$. A termotolerância é ainda um dos fatores que contribuem com a alta patogenicidade da espécie, sendo a maior causadora de casos clínicos em pinguins ${ }^{(6,27)}$, os quais apresentam uma temperatura corporal de cerca de $40{ }^{\circ} \mathrm{C}^{(2)}$. $\mathrm{O}$ pH das amostras de água coletadas variou de 7 a 9 e não influenciou significativamente $(\mathrm{p}>0,05)$ no isolamento do gênero Aspergillus, bem como da espécie A. fumigatus. Este gênero fúngico foi isolado em 100\% (2/2) das amostras de água com pH 9, em 83,3\% (15/18) com pH 7, e em 73,7\% (14/19) com $\mathrm{pH} 8$; em uma amostra não foi possível fazer a avaliação do $\mathrm{pH}$.

Essa taxa similar de isolamento de fungos do gênero Aspergillus encontrada em distintos valores de $\mathrm{pH}$ das amostras de água pode ser explicada pelo fato de que este gênero fúngico é capaz de regular o seu $\mathrm{pH}$ interno, independente do ambiente externo, podendo habitar ambientes de $\mathrm{pH}$ ácidos a alcalinos, com variação de 3,5 a $9^{(28,29)}$. Há poucos estudos na literatura que analisam a variação do $\mathrm{pH}$ em A. fumigatus; entretanto, autores concordam que a espécie deve apresentar regulações internas semelhantes à de outras espécies do gênero ${ }^{(30-32)}$.

Em relação às estações do ano, das 40 amostras analisadas, Aspergillus spp. foi isolado em 66,7\% (2/3), 69,2\% (9/13), 76,9\% (10/13) e 100\% (11/11) das amostras colhidas no verão, outono, inverno e na primavera, respectivamente, sem apresentar diferença estatística significativa $(\mathrm{p}>0,05)$. No estudo realizado por Mezzari et al. ${ }^{(33)}$, a estação que apresentou maior concentração de Aspergillus spp. no ambiente foi o verão, principalmente pelo aumento da temperatura, condição que permite um maior desenvolvimento desses microrganismos ${ }^{(34-36)}$; no entanto, no presente estudo, a sazonalidade não influenciou no isolamento deste gênero fúngico, embora o número de amostras coletadas no período de verão tenha sido escasso em relação às demais estações, devido a uma limitação referente ao período de realização do trabalho.

Com relação especificamente ao isolamento da principal espécie patogênica, A. fumigatus, a sazonalidade influenciou significativamente $(\mathrm{p}<0,001)$, sendo que na primavera e no inverno obtiveram-se os maiores números de amostras positivas, $100 \%$ e $76,9 \%$, respectivamente; enquanto que no verão nenhuma amostra foi positiva e no outono houve $30,8 \%$ de positividade. Esse alto nível de significância encontrado quanto à influência da sazonalidade no isolamento da espécie $A$. fumigatus pode ser justificado pela variação das condições ambientais ao longo das estações na cidade de Rio Grande, RS, Brasil. A alta positividade encontrada na primavera e no inverno pode estar relacionada a boas condições ambientais para o desenvolvimento desses fungos, como o aumento dos ventos, que carreiam partículas em suspensão, aumentando a quantidade de matéria orgânica nos tanques; o aumento da temperatura na primavera, bem como o aumento de chuvas no inverno, o que eleva a umidade ${ }^{(37-41)}$.

Durante o período de estudo, o número de pinguins em reabilitação no tanque variou de três a onze, sendo que $70 \%$ (14/20) das amostras coletadas em período de densidade populacional de até cinco animais foram positivas para o isolamento de fungos do gênero Aspergillus, aumentando para $90 \%$ (18/20) de positividade em períodos com seis a onze pinguins. Embora essa diferença de positividade para o gênero Aspergillus não tenha sido significativa ( $\mathrm{p}=0,114)$, a densidade populacional influenciou significativamente no isolamento da espécie $A$. fumigatus, sendo que a taxa de positividade foi de 90\% (18/20) em períodos de alta densidade populacional e de apenas 35\% (7/20) em períodos com poucos pinguins em reabilitação no tanque $(\mathrm{p}<0,001)$.

Ressalta-se que as estações de primavera e inverno, em que se obteve maior isolamento de $A$. fumigatus, $81,8 \%(18 / 22)$ e $69,2 \%$ (18/26), respectivamente, também foram os períodos com maior densidade populacional no Centro. No outono, apenas $15,4 \%(4 / 26)$ e no verão nenhuma (0/6) das coletas ocorreu em períodos com mais de cinco pinguins no tanque $(\mathrm{p}<0,001)$.

De acordo com Asan et al. ${ }^{(42)}$, os fungos do gênero Aspergillus, especificamente a espécie A. fumigatus, são seres sapróbios, que utilizam diversos tipos de matéria orgânica para sua nutrição. Os resultados observados na análise do parâmetro densidade de pinguins corroboram com essa afirmação. Com o 
aumento de pinguins, há o claro aumento da matéria orgânica em suspensão na água, o que facilita o desenvolvimento desses fungos.

\section{Conclusões}

Este estudo demonstrou que os fungos do gênero Aspergillus e a espécie A. fumigatus estão presentes na água onde os pinguins permanecem para reabilitação, que pode ser uma potencial fonte de infecção para os animais debilitados e/ou predispostos ao desencadeamento da doença. Estes fungos são detectados principalmente pela incubação a $37^{\circ} \mathrm{C}$ e o aumento da matéria orgânica favorece a sua proliferação na água.

\section{Referências}

1. Flach EJ, Stevenson MF, Henderson GM. Aspergillosis in gentoo penguins (Pygoscelis papua) at Edinburgh Zoo, 1964 to 1988. Veterinary Record. 1990;126:81-85.

2. Abundis-Santamaria E. Aspergillosis in birds of prey. 2003. Available at: http//www.aspergillus.man.ac.uk. Acesso em abril 2015

3. Park S, Mehrad B. Innate immunity to Aspergillus species. Clinical Microbiology Reviews. 2009;22:535551.

4. Ainsworth GC, Rewell R. The incidence of aspergillosis in captive wild birds. Journal of Comparative Pathology. 1949;59:213-224.

5. Khan Z, Pal M, Paliwal DK, Damodaran VN. Aspergillosis in imported penguins. Sabouraudia. 1977;v. 15:p. 43-45.

6. Xavier M, Soares MP, Meinerz AR, Nobre MO, Silva-Filho RP, Osório LG, et al. Aspergillosis: a limiting factor during recovery of captive magellanic penguins. Braz J Microbiol. 2007;v. 38:p. 480-484.

7. Waeyenberghe LV, Pasmans F, Beernaert LA, Haesebrouck F, Vercammen F, Verstappen F, et al. Microsatellite typing of avian clinical and environmental isolates of Aspergillus fumigatus. Avian Pathology. 40:73-77.

8. Xavier MO, Leite AT, Soares MP, Osório LG, Martins A, Albano AP, et al. Aspergilose em Pingüim-demagalhães (Spheniscus magellanicus) Relato de Caso. Veterinária e Zootecnia. 2006;13:28-32.

9. Ruoppolo V, Adornes A, Nascimento A, Silva-Filho R. Reabilitação de pingüins afetados por petróleo. Clín. Vet. 2004;51:78-83.

10. Graczyk TK, Cranfield MR. Maternal transfer of anti-Aspergillus spp. Immunoglobulins in African Blackfooted Penguins (Spheniscus demersus). Journal of Wildlife Diseases. 1995;31(4):545-549.

11. Santos LP dos, Gunski RJ. Revisão de dados citogenéticos sobre a avifauna brasileira. Revista Brasileira de Ornitologia. 2006;14(1):35-45.

12. Brandão ML, Braga KM, Luque JL. Marine debris ingestion by Magellanic penguins, Spheniscus magellanicus (Aves: Sphenisciformes), from the Brazilian coastal zone. Marine pollution bulletin. 2011;62(10):2246-9.

13. Jehl JR. Mortality of magallanic penguins in Argentina. The Auk. 1975;92(3):596-598.

14. Randall RM, Randall BM, Bevan J. Oil pollution and penguins - Is cleaning justified? Marine Pollution Bulletin. 1980;11(8):234-237.

15. García-Borboroglu P, Boersma P, Ruoppolo V, Reyes L, Rebstock GA, Griot K, et al. Chronic oil pollution harms Magellanic penguins in the Southwest Atlantic. Mar. Pollut. Bull., 52, 193-198. 2006;52:193-198.

16. Mazet JA, Newman SH, Gilardi KV, Tseng FS, Holcomb JB, Jessup DA, et al. Advances in Oiled Bird Emergency Medicine and Management. Journal of Avian Medicine and Surgery. 2002;16(2):146-149.

17. Mäder A, Sander M, Junior GC. Ciclo sazonal de mortalidade do pinguim-de-magalhães, Spheniscus 
magellanicus, influenciado por fatores antrópicos e climáticos na costa do Rio Grande do Sul, Brasil. Revista Brasileira de Ornitologia. 18(3):228-233.

18. Girardin H, Sarfati J, Traore F, Dupouy CJ, Derouin F, Latge JP. Molecular epidemiology of nosocomial invasive aspergillosis. Journal Clinical Microbiology. 1994;32:684-690.

19. Chazalet V, Debeaupuis JP, Sarfati J. Molecular typing of environmental and patients isolates of Aspergillus fumigatus from various hospital settings. Journal Clinical Microbiology. 1998;36:1494-1500.

20. Vorbeck-Meister I, Sommer R, Vorbeck F, Hörl WH. Quality of water used for haemodilysis: bacteriological and chemical parameters. Nephrology Dialysis Transplantation. 1999;14:666-675.

21. Warris A, Gaustad P, Meis MJ, Voss A, Verweij EP, Abrahamsen GT. Recovery of filamentous fungi from water in a pediatric bone marrow transplantation unit. The Journal Hospital Infection. 2001;47:143-148.

22. Varo SD, Henrique C, Martins G, Jorge M, Cardoso DO, Sartori FG, et al. Isolamento de fungos filamentosos em água utilizada em uma unidade de hemodiálise. Journal of Nephrology. 2007;40(3):326-331.

23. Gercovich F, Richman SP, Rodriguez V, Luna M, Mccardie KB, Bodey GP. Successful control of systemic Aspergillus niger infection in two patients with acute leukemia. Cancer. 1975;36(6):2271-2276.

24. Anaissie EJ, Stratton SL, Dignani MC, Summerbell RC, Rex JH, Monson TP, et al. Aspergillus species recovered from a hospital water system: a 3-year prospective study. Clinical Infections Diseases. 2002;34:780789.

25. Latge J. Aspergillus fumigatus and Aspergillosis. Clinical Microbiology Reviews. 1999;12(2):310-350.

26. Gomes E, Guez MA, Martin N, Silva RD. Enzimas termoestáveis: fonte, produção e aplicação industrial. Química nova. 2007;30:136-145.

27. Boff C, Brun CP, Miron D, Zoppas BC, Pasqualotto AC. The effect of different incubation temperatures on the recovery of Aspergillus species from hospital air. American Journal of Infection Control. 2012;40(10):10161017. Available at: http://dx.doi.org/10.1016/j.ajic.2012.01.029. Acesso em abril 2015

28. Arst HN, Penãlva MA. pH regulation in Aspergillus and parallels with higher eukaryotic regulatory systems. Trends in genetics. 2003;19(4):224-231.

29. Eisendle M, Oberegger H, Buttinger R, Eisendle M, Oberegger H, Buttinger R, et al. Biosynthesis and Uptake of Siderophores Is Controlled by the PacC-Mediated Ambient-pH Regulatory System in Aspergillus nidulans. Eukaryot Cell. 2004: 561-563

30. Peñalva MA, Jr HN, Pen MA, Arst HN. Regulation of Gene Expression by Ambient pH in Filamentous Fungi and Yeasts. 2002;66(3).

31. Bignell E. The Aspergillus $\mathrm{pH}-$-responsive transcription factor PacC regulates virulence. Mol. Microbiol. 2005;55:1072-1084.

32. Peñalva MA, Tilburn J, Bignell E, Jr HN. Ambient $\mathrm{pH}$ gene regulation in fungi : making connections. Trends in Microbiology. 2008;(May):291-300.

33. Mezzari A, Perin C, Santos Júnior S, Bernd L. Airborne fungi in the city of Porto Alegre, Rio Grande do Sul, Brazil. Rev. Inst. Med. Trop. 2002;44(5):269-272.

34. Khulbe R, Drugapal A. Population dynamics of Geofungi in a polluted freshwater body at Nainital, Kumaun Himalaya. Poll. Res. 1992:213-219.

35. Bhat S, Kamili A. A Preliminary Aquatic Mycological Study of Dal Lake. J. of Res. And develop. 2004;4:8795.

36. Sharma K, Parveen S. Ecological Study of Fungi Isolated from the Surface Water of Dudhawa Dam Dhamtari, Chhattisgarh. India Journal of Phytology. 2011:06-08.

37. Gambale W, Purchio A, Paula C. Influência de fatores abióticos na dispersão aérea de fungos na cidade de São Paulo, Brasil. Rev. Microbiol. 1983;14(3):204-214.

38. Hyvärinen A, O'rourke M, Meldrum J, Stetzenbach L. Influence of cooling type on airborne viable fungi. J. Aer. Sci. 1995;28(1):887-888.

39. Mehdi FS, Saifullah SM. Species diversity and seasonal occurrence of fungi on seedlings of Avicennia marina (Forsk.) Vierh. Pakistan Journal of Biological Sciences. 2000. 
40. Távora L, Gambale W, Heins-Vaccari E, Arriagada GLH, Lacaz CS, Santos CR, et al. Comparative performance of two air samplers for monitoring airborne fungal propagules. Braz. J. Med. Biol. Res. 36(613616):2003.

41. Instituto Nacional de Metereologia (INMET) [Internet]. Monitoramento climático. Dec 2013 [cited 2014 Jan 03]. Available at: http://www.inmet.gov.br/portal/. Acesso em abril 2015

42. Asan A, Kirgi Z, Sen B, Elipek BC, Guner U, Guher H. Isolation, identification and seasonal distribution of airborne and waterborne fungi in Terkos Lake (Istanbul-Turkey). J. Basic Microbiol. 2003:83-95. 